\title{
Bacterial growth and toxin production in ileostomy effluents
}

\author{
D. A. A. ALA ALDEEN and *M. R. BARER
}

Department of Clinical Tropical Medicine, The London School of Hygiene and Tropical Medicine, Keppel Street, London WC1E $7 H T$

\begin{abstract}
Summary. Escherichia coli (2), Vibrio cholerae (2) and Aeromonas sobria (1) strains were examined for their ability to grow and produce toxins in samples of ileostomy fluid. Three categories of response were observed: no detectable growth, growth without detectable toxin, and growth with detectable toxin. Clear differences were apparent between samples of ileostomy fluid obtained from different individuals and between samples obtained from the same individual at different times. The patterns of response were unique for each of the five test strains. We propose that the procedure developed forms a basis for investigating the host-parasite relationship in diarrhoeal disease.
\end{abstract}

\section{Introduction}

Many bacterial species are believed to cause diarrhoea by growing and producing toxins in the small intestine. In assessing the potential role of an individual isolate as an enteropathogen it is conventional for toxin production to be determined by means of standard in-vitro tests (Wachsmuth, 1984). The degree to which these procedures reflect the ability of the test organism to produce the toxin concerned in the relevant conditions in vivo is unknown.

Nutritional and other environmental conditions exert a profound influence on bacterial growth and toxin production; e.g., production and release of Escherichia coli heat-labile toxin (LT) is affected by the amino acid, carbon source, oxygen and protease content of the growth medium as well as by temperature and $\mathrm{pH}$ (Gilligan and Robertson, 1979; Kunkel and Robertson, 1979). It has also been shown that different biotypes of Vibrio cholerae require different conditions for optimal toxin production (Kusama and Craig, 1970).

Notwithstanding such data from in-vitro studies, almost nothing is known about the local environmental influences that may be critical in determining whether virulence factors are expressed in the human small intestine. To address one aspect of this problem, we devised a technique in which

Received 22 Aug. 1988; accepted 10 Oct. 1988.

*Correspondence should be sent to: Dr M. R. Barer, Department of Microbiology, University of Newcastle upon Tyne Medical School, Framlington Place, Newcastle upon Tyne NE2 4HH. growth and toxin production by bacterial enteropathogens may be measured in samples of ileostomy fluid.

\section{Materials and methods}

\section{Ileostomy fluid samples}

Samples were obtained from four subjects. Samples 13 were from otherwise healthy individuals who had had ileostomies fashioned at least 2 years earlier after resections for ulcerative colitis. All three were on normal diets and were not taking any drugs. Samples $4 a-4 d$ were serial specimens obtained on consecutive days from an in-patient who had been treated for a pulmonary infection and who had had a colectomy for Crohn's disease 18 years earlier. She had stopped antibiotic therapy 3 days before the first sample was collected. All samples were extruded into sterile plastic containers directly from ileostomy bags as soon as feasible after bowel movements. They were stored at $-20^{\circ} \mathrm{C}$ from the day of collection until further processing.

\section{Preparation of ileostomy samples}

After careful homogenisation, each sample was divided into $1-\mathrm{ml}$ aliquots, each of which was mixed with an equal volume of sterile distilled water. The pre-existing bacterial flora was then suppressed by exposing these mixtures to microwave irradiation (30-45 s) in a Phillips M710 microwave oven at setting 5 (approx. $350 \mathrm{~W}$ ). Thereafter $0.03-\mathrm{ml}$ samples were plated on to nutrient agar and gave no visible bacterial growth after incubation at $37^{\circ} \mathrm{C}$ for $18 \mathrm{~h}$. 


\section{Bacterial strains}

Two strains of $E$. coli, one (SIEc1) producing heatlabile toxin (LT) and the other (HWSEc4) producing Vero toxin (VT), and two strains of $V$. cholerae 01 (SIVc3 and SIVc4) producing cytotoxin (CYT) and enterotoxin (CT) respectively, and a cytotoxin (ACT)-producing strain of Aeromonas sobria (SMAs27) were used. All except strain SIVc3 (an environmental isolate) were clinical isolates from patients with diarrhoea.

\section{Cultural conditions}

The test organisms were inoculated into 2-ml volumes of Tryptone Soy Broth (Oxoid) supplemented with yeast extract (Oxoid) $1.2 \% \mathrm{w} / \mathrm{v}$ (TSBYE). After overnight incubation at $37^{\circ} \mathrm{C}$, the culture was diluted to give an optical density of $0 \cdot 1$ at $540 \mathrm{~nm} ; 10 \mu$ l of diluted culture was then inoculated into each pretreated ileostomy fluid sample and into $2 \mathrm{ml}$ of TSBYE as a positive control. In the case of the CT-producing strain of $V$. cholerae, Syncase medium (Finkelstein et al., 1966) was used in place of TSBYE. Uninoculated aliquots of each ileostomy-fluid sample were incubated and treated as negative controls. The samples and controls were then incubated at $37^{\circ} \mathrm{C}$ for $48 \mathrm{~h}$ in a shaking water bath $(150 \mathrm{rpm})$. Thereafter the samples were carefully mixed in a Colworth stomacher and the numbers of cfu determined (Miles et al., 1938). Every sample was cultured on Columbia agar and an appropriate selective medium $(E$. coli and $A$. sobria on MacConkey's agar and $V$. cholerae on TCBS). Uninoculated control samples were subcultured on to all three culture media. All media were obtained from Oxoid. The remainder of the sample was then centrifuged at $14000 \mathrm{rpm}$ in an Eppendorf 5413 centrifuge for $25 \mathrm{~min}$ and the supernate removed for toxin testing.

\section{Toxin testing}

Toxicity was determined in cell culture with Vero cells as previously described (Barer et al., 1986). Briefly, established monolayers of Vero cells were exposed to serial two-fold dilutions of the test material and inspected for a cytopathic effect (CPE) after incubation for $20 \mathrm{~h}$ for CYT, CT, ACT, and LT and after $44 \mathrm{~h}$ for VT. Previous studies have shown that all five CPEs are readily discernible and quantifiable in this assay (Giugliano et al., 1982; Barer et al., 1986). Titres refer to the last dilution showing a specific CPE; this represents the final dilution of the starting material as used in the assay.

\section{Results}

Measurements of bacterial growth and toxin production in each of the four ileostomy fluids from different patients and in serial samples from the same patient are shown in tables I and II. Significant growth of the inoculated organism occurred in 19 of the 35 different permutations of organism and test sample. None of the samples supported growth of all five strains to the same extent as control medium. However, in five instances higher colony counts than control were obtained-strain SIVc3 in sample 3, strain SIEcl in samples 1, 2, and 4a, and strain SMAs27 in sample 4a. Overall, counts obtained for $A$. sobria and $V$. cholerae were consistently lower than those obtained for the two E. coli strains. Results with samples 4 a-d (table II) suggested decreased growth of the inoculated organisms within the serial ileostomy fluids.

Despite achieving substantial suppression, exposure of the samples to microwave irradiation did not completely eliminate subsequent growth of the pre-existing flora. Bacterial counts of $2 \cdot 7-7 \cdot 7 \log _{10}$ $\mathrm{cfu}$ were detected in control samples inoculated on to Columbia agar. However, bacterial numbers in these samples were considerably lower and often undetectable on the selective media and, where

Table I. Growth and toxin titres of test strains in ileostomy-fluid samples from different individuals

\begin{tabular}{|c|c|c|c|c|c|c|c|c|c|c|}
\hline \multirow{4}{*}{$\begin{array}{c}\text { Strain } \\
\text { designation }\end{array}$} & \multicolumn{10}{|c|}{ Viable count and toxin production in } \\
\hline & \multicolumn{8}{|c|}{ ileostomy sample no. } & & \\
\hline & \multicolumn{2}{|c|}{1} & \multicolumn{2}{|c|}{2} & \multicolumn{2}{|c|}{3} & \multicolumn{2}{|c|}{$4 a$} & \multicolumn{2}{|c|}{$\begin{array}{l}\text { control } \\
\text { medium }\end{array}$} \\
\hline & count* & titre & count & titre & count & titre & count & titre & count & titre \\
\hline SMAs 27 (ACT) & $5 \cdot 7$ & 0 & $6 \cdot 7$ & 0 & $6 \cdot 5$ & 0 & $8 \cdot 8$ & 0 & $8 \cdot 1$ & 48 \\
\hline SIVc4 (CT) & $<2.7$ & 0 & $<2.7$ & 0 & $6 \cdot 4$ & 192 & $<2 \cdot 7$ & 0 & $8 \cdot 0$ & 2400 \\
\hline SIVc3 (CYT) & $<2 \cdot 7$ & 0 & $<2 \cdot 7$ & 0 & $6 \cdot 1$ & 12 & $<2 \cdot 7$ & 0 & $4 \cdot 4$ & 48 \\
\hline SIEc1 (LT) & $9 \cdot 3$ & 0 & $9 \cdot 0$ & 48 & $7 \cdot 3$ & 0 & $9 \cdot 0$ & 96 & $8 \cdot 2$ & 192 \\
\hline HWSEc4 (VT) & 6.5 & 192 & $5 \cdot 2$ & 96 & $7 \cdot 2$ & 24 & 8.7 & 24500 & $9 \cdot 2$ & 24500 \\
\hline
\end{tabular}

* $\log _{10} \mathrm{cfu} / \mathrm{ml}$ determined by surface viable count on the appropriate selective medium. 
Table II. Growth and toxin titres of test strains in serial daily (a-d) ileostomy samples from subject 4

\begin{tabular}{|c|c|c|c|c|c|c|c|c|c|c|}
\hline \multirow{4}{*}{$\begin{array}{c}\text { Strain } \\
\text { designation }\end{array}$} & \multicolumn{10}{|c|}{ Viable count and toxin production in } \\
\hline & \multicolumn{8}{|c|}{ ileostomy sample no. } & \multirow{2}{*}{\multicolumn{2}{|c|}{$\begin{array}{l}\text { control } \\
\text { medium }\end{array}$}} \\
\hline & \multicolumn{2}{|c|}{$4 a$} & \multicolumn{2}{|c|}{$4 b$} & \multicolumn{2}{|c|}{$4 c$} & \multicolumn{2}{|c|}{$4 d$} & & \\
\hline & count* & titre & count & titre & count & titre & count & titre & count & titre \\
\hline SMAs27 (ACT) & $8 \cdot 8$ & 0 & $<2 \cdot 7$ & 0 & $<2 \cdot 7$ & 0 & $<2.7$ & 0 & $8 \cdot 1$ & 48 \\
\hline SIVc4 (CT) & $<2 \cdot 7$ & 0 & $<2 \cdot 7$ & 0 & $<2 \cdot 7$ & 0 & $<2 \cdot 7$ & 0 & $8 \cdot 0$ & 2400 \\
\hline SIVc3 (CYT) & $<2 \cdot 7$ & 0 & $<2 \cdot 7$ & 0 & $<2 \cdot 7$ & 0 & $<2 \cdot 7$ & 0 & $4 \cdot 4$ & 48 \\
\hline SIEcl (LT) & $9 \cdot 0$ & 96 & $7 \cdot 6$ & 3 & $3 \cdot 8$ & 3 & $3 \cdot 4$ & 0 & $8 \cdot 2$ & 192 \\
\hline HWSEc4 (VT) & $8 \cdot 7$ & 24500 & $7 \cdot 0$ & 1536 & $4 \cdot 0$ & 6 & $<2.7$ & 0 & $9 \cdot 2$ & 24500 \\
\hline
\end{tabular}

${ }^{*} \log _{10} \mathrm{cfu} / \mathrm{ml}$ determined by surface viable count on the appropriate selective medium.

present, the indigenous bacteria showed clearly different colonial characteristics from the inoculated test bacteria and culture on selective media gave counts less than 100 -fold of the count recorded for the test organism.

Growth of the enteropathogen was associated with toxin activity on twelve occasions but levels of bacterial growth and toxin activity were not correlated: in seven instances growth without detectable toxin production was recognised; variable toxin titres were seen without corresponding growth yields.

An intrinsic CPE (titre $\leqslant 12$ ) was observed in uninoculated ileostomy-fluid samples which was readily distinguishable from the CPEs due to specific toxins. The effect resembled that previously observed after exposure of tissue cells to bile.

To determine whether the ileostomy-fluid samples had any direct effect on toxin activity, an equal volume of control medium preparation was mixed with each of the samples and, in every case, the titre was reduced two-fold which was consistent with the dilution factor.

Individual ileostomy samples were heterogenous with respect to their growth supporting properties and associated toxin activity. Serial samples provided by subject 4 showed that such variation could occur with time in the same individual (see table II).

\section{Discussion}

This study has demonstrated that the growth and toxin production by several enteropathogens can be measured in ileal effluents. Although the full significance of these findings requires further evaluation, the range of responses obtained clearly suggests that important determinants involved in the pathogenesis of intestinal disease may be profoundly affected by the composition of ileostomy fluid. Three categories were observed: undetectable growth, growth without toxin production, and growth with toxin production. When present, toxin activity was usually lower than that found after growth in a standard culture medium.

The differences in composition of the ileostomy samples responsible for our results were not determined and can only be the subject of speculation; four possibilities exist-differences in diet, digestive secretions, immune status and the preexisting microbial flora.

The potential importance of such studies is highlighted by the finding that samples from one individual changed from being relatively permissive for growth and toxin production into a hostile non-permissive environment from one day to the next. This finding and the prospect of identifying the factors responsible raises the possibility that the small intestinal environment can be specifically manipulated to resist symptomatic enteric infections.

The five bacterial strains examined in this study showed different patterns of toxin activity. Our results show that it is possible to rank the enteropathogenicity of the strains examined as HWSEc4 (VT) $>$ SIEc1 (LT) $>$ SIVc4 (CT) $>$ SIVc3 $(\mathrm{CYT})>$ SMAs27 (ACT). In this regard it is interesting to note that asymptomatic infections are common in outbreaks of cholera (Gangarosa and Mosley, 1970) while recent human volunteer studies with Aeromonas spp. yielded a very low frequency of symptoms (Morgan et al., 1985).

We do not claim that ileostomy samples examined under our test conditions reflect all of the 
elements influencing the expression of bacterial virulence in the small intestine. Clearly there are many important differences between this material and normal ileal fluid. Nevertheless, the samples examined reflect individual differences (both shortand long-term) that may reasonably be expected to occur in the ileal contents of normal individuals. Therefore, our test system may be considered as a practical compromise through which the further investigation of an important area of bacterial pathogenesis may become feasible. Our experiments have demonstrated differences between

\section{REFERENCES}

Barer M R, Millership S E, Tabaqchali S 1986 Relationship of toxin production to species in the genus Aeromonas. Journal of Medical Microbiology 22: 303-309.

Finkelstein R A, Atthasampunna P, Chulasamaya M, Charunmethee P 1966 Pathogenesis of experimental cholera: biological activities of purified procholeragen A. Journal of Immunology 96: 40-49.

Gangarosa E J, Mosley W H 1974 Epidemiology and surveillance of Cholera. In: Barua D, Burrows W (eds) Cholera. WB Saunders, Philadelphia, pp 381-403.

Gilligan P H, Robertson D C 1979 Nutritional requirements for synthesis of heat-labile enterotoxin by enterotoxigenic strains of Escherichia coli. Infection and Immunity 23: 99107.

Giugliano L G, Mann G F, Drasar B S 1982 Response of mammalian cell lines to the toxins of Escherichia coli. Journal of Medical Microbiology 15: 531-539. ileostomy samples with respect to their ability to support growth and toxin production by putatative enteropathogens. In addition, we suggest that the approach established is a practical means by which aspects of host susceptibility to enteric infections and previously unidentified bacterial virulence factors may be further investigated.

We thank the Ileostomy Association of Great Britain and Ireland for their invaluable assistance, Anita Singh for technical support, the Humane Research Trust (Cheshire) for financial assistance, and Drs G. T. Macfarlane, B. S. Drasar, N. McNeil, and Professor M. Sussman for their help.

Kunkel S L, Robertson D C 1979 Factors affecting the release of heat-labile enterotoxin by enterotoxigenic Escherichia coli. Infection and Immunity 23: 652-659.

Kusama H, Craig J P 1970 Production of biologically active substances by two strains of Vibrio cholerae. Infection and Immunity 1 : 80-87.

Miles A A, Misra S S, Irwin J O 1938 The estimation of the bactericidal power of the blood. Journal of Hygiene 38 : $732-$ 749.

Morgan D R, Johnson P C, DuPont H L, Satterwhite T K, Wood L V 1985 Lack of correlation between known virulence properties of Aeromonas hydrophila and enteropathogenicity for humans. Infection and Immunity 50: 6265.

Wachsmuth K 1984 Laboratory detection of enterotoxins. In: Ellner P D (ed) Infectious diarrheal disease. Marcel Dekker Inc., New York, pp 93-116. 